\title{
Ultrasound Doppler Array Velocimeter Used for Vortex Investigations of Liquid Metals
}

\author{
Franke, Sven; Büttner, Lars; Czarske, Jürgen; Eckert, Sven*
}

Laboratory of Measurement and Testing Technologies, Faculty of Electrical Engineering and Information Technology, Technische Universität Dresden, Helmholtzstrasse 18, 01069, Dresden, Germany

* Department Magnetohydrodynamics, Institute Safety Research, Forschungszentrum DresdenRossendorf, Bautzner Landstraße 128, 01328, Dresden, Germany

An ultrasound Doppler measurement system for measuring the flow velocity field of magnetically driven liquid metal is presented: Two orthogonally arranged ultrasound Doppler sensor line arrays each with 25 piezo elements allow the two dimensional measurement of two flow velocity components $(2 \mathrm{~d} / 2 \mathrm{c})$ in an area of $70 \times 70 \mathrm{~mm}^{2}$. To obtain a high spatial and a high temporal resolution, a specific multiplex mode for controlling the line array elements is used. First measurement results of liquid metal flows driven by a rotating magnetic (RMF) field are shown.

\section{Introduction}

Magnetohydrodynamics provides manifold possibilities for electromagnetic flow control in industrial processes. The purpose of such investigations is to manipulate flows of liquid metals, semiconductors or electrolytes in fields of metallurgy, crystal pulling and electrochemistry by means of magnetic forces, in order to optimize production processes and to improve product qualities. Particularly the impact of magnetic fields on convection, mass transport, heat transmission and solidification of liquid metals and the resulting material properties are determined $[1,2]$.

Typical application fields of magnetohydrodynamics in industry can be found in the fabrication of monocrystalline semiconductor crystals according to the Czochralski process [3] and in continuous casting method at steel fabrication [4]. However, many processes in magnetohydrodynamics, e.g the flow characteristic of steel melt influenced by different magnetic fields in the mold at continous steel casting, are not yet exactly known.

Hence, fundamental researches are performed in both theoretically-numeric and experimental methods. For experimental investigations alloys with very low melting points are used to realize model experiments at technically feasible temperatures. In this manner, for example, liquid Gallium Indium Tin (GalnSn) is utilized for magnetohydrodynamic flow experiments at room temperature [1].

Adequate non-invasive, multidimensional, multi-component measurements of flow fields are desired to verify these experiments. Often optical methods of flow measurement technique like Particle Image Velocimetry (PIV) and Laser Doppler Anemometry (LDA) are applied [5]. However, these techniques can not be used with liquid metals due to their opacity. In that case, particularly ultrasound based methods like pulsed wave Ultrasound Doppler Velocimetry (UDV) [1,2] can be applied. The principle of pulsed wave UDV is based on the repetitive emmission of short ultrasound pulses and the reception of echo signals scattered by seeding particles similar to the method of sonar. By correlating two sequenced echo signals the velocity profile can be determined.

\section{State-of-the-Art and Motivation}

For flow researches a number of commercial instruments for Ultrasound Doppler Velocimetry (UDV) are available on the market providing a measurement of one velocity component along a line or two or three velocity components simultaneously at one point. Possibilities for areal measurements of two velocity components $(2 \mathrm{~d} / 2 \mathrm{c})$ are limited by the maximum number of ultrasound transceivers which can be used at one velocimeter (e.g. 10 with the DOP2000 from Signal Processing, Lausanne, Switzerland [6]). A higher number of scanning lines necessary for present investigations of flow characteristics in magneticallydriven liquid metals can be realized with commercial instruments only by a time-consuming mechanical traversing of ultrasound sensors [7].

Therefore, an pulsed wave ultrasound Doppler measurement system providing a $2 \mathrm{~d} / 2 \mathrm{c}$ flow field measuring with high temporal and spatial resolution is under development. 


\section{Measurement System}

\subsection{Design of the Ultrasound Transducer Line Array}

The ultrasound Doppler measurement system includes the application of monolithic arrays of ultrasound transducers. This allows an electronic traversing resulting in a faster scanning of flow fields compared to the mechanical traversing technique.

For the measurement system two identical ultrasound transducer line arrays, each with 25 transducer elements, are arranged orthogonally to each other allowing the $2 \mathrm{~d} / 2 \mathrm{c}$ flow field measurement in a square plane of $70 \times 70 \mathrm{~mm}^{2}$ (Fig. 1). The design of the line arrays is determind by special marginal conditions as follows.

To achieve a high spatial resolution the transmission frequency of an ultrasound transducers is supposed to be as high as possible. In detail a high transmission frequency corresponds to a short wavelength and results in an ultrasound beam with low beam divergence and a high lateral resolution. The axial resolution is improved as well due to the shorter ultrasound pulse. However, the upper limit of the ultrasound frequency is determined by the limited frequency range of necessary electronic components such as high voltage switches and losses of sound power in liquid metal due to higher acoustic absorption at higher frequencies. The bandwidth of the transducers is required be to as high as possible resulting in shorter ultrasound pulses and so in a higher axial resolution. Hence, the piezo elements were designed with a center frequency of around $8 \mathrm{MHz}$ at a bandwidth of about $2.6 \mathrm{MHz}$.

Contrary demands have to be considered for the size of the piezo elements of the array. A scanning of flow velocity fields with a small lateral scan step width necessitates a small size of these elements. However, a low divergence of the ultrasound beam requires an edge length of a piezo element, considering a square piezo transducer, much bigger than the ultrasound wavelength [8]. To comply with both requirements, a small lateral step width for scanning and a low divergence of the ultrasound beam, a special mode of operation is introduced. Instead of using square piezo elements for the line array, rectangular elements with a width half of the height are applied bisecting the lateral step width. For the present design the dimension of $2.5 \times 5 \mathrm{~mm}^{2}$ per piezo element was chosen. In operation two neighboring rectangular piezo elements are combined to a pair acting as one square ultrasound transducer with an acitive area of $5 \times 5 \mathrm{~mm}^{2}$ which can be shifted by half of its edge length. By using this method the 25 piezo elements of the line arrays result in 24 applicable transducer pairs. The line array structure is shown in Fig. 2. The sensitive array length is $70 \mathrm{~mm}$ due to gaps between the separate piezo elements caused by the production process.

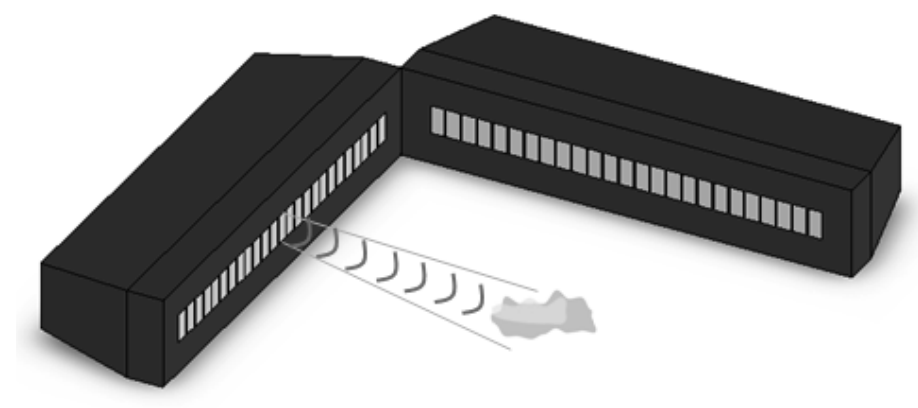

Figure 1. Two monolithic ultrasound line arrays arranged orthogonally for $2 \mathrm{~d} / 2 \mathrm{c}$ flow field measurements

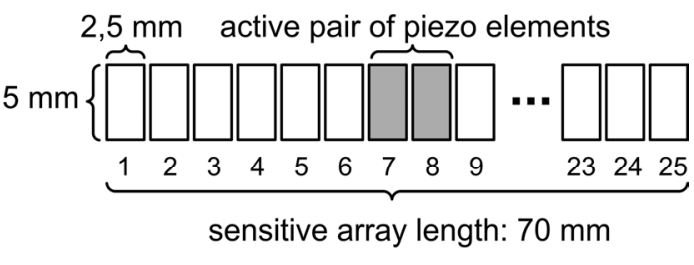

Figure 2. Design of the ultrasound line array

\subsection{Operation Principle and Driver Electronics}

The demand for a high spatial and a high temporal resolution (i.e. high frame rate) of the measurement system are complementary to each other. This results from an increasing measurement duration with a growing number of array elements when capturing one whole flow field in time division mode.

To achieve a high temporal resolution notwithstanding a modified time division scheme (Fig. 3) for controlling the ultrasound transducer is used in which four piezo pairs transmit and receive in parallel reducing the measurement duration by factor 4 . Thereby, the spacing between the acitve piezo pairs is chosen in such a manner that there is only a negligible crosstalk. The time division scheme is realized in such a way, that no piezo element is sending and receiving on two sequent time steps to prevent a distortion of echo signal as a result of multiple echos. 


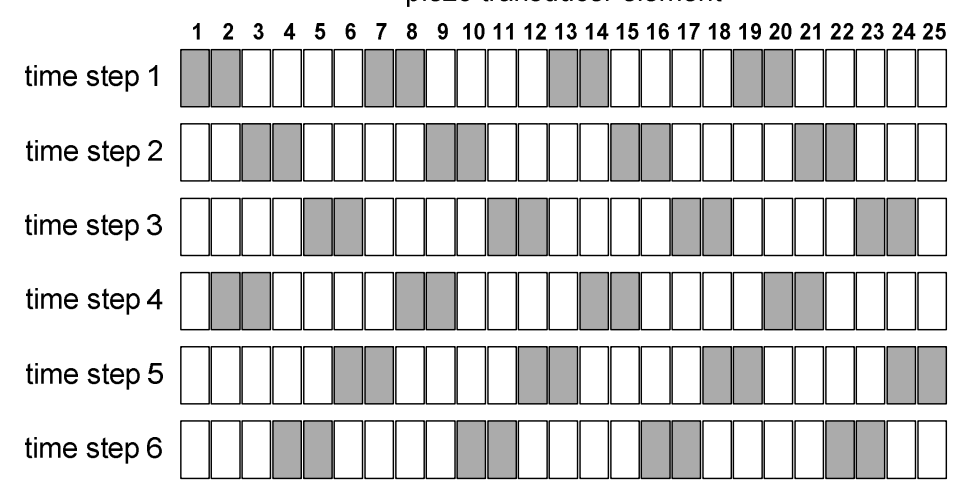

active piezo elements inactive piezo elements

Figure 3. Multiplex scheme for parallel time division in 4-channel operation mode

The overall 24 transducer pairs can scan the whole flow field in $n=6$ steps assuming a parallel operation mode of four piezo pairs. It is shifted to the particular next scanning step after the echo signal of the maximum measurement depth of the recently active transducers has been received.

Using GalnSn $\left(c=2747 \mathrm{~m} \cdot \mathrm{s}^{-1}\right)$ as liquid metal a measurement depth of $d=70 \mathrm{~mm}$ corresponds to a time interval of $\mathrm{T}=2 \mathrm{~d} / \mathrm{c}=2 \cdot 0.07 \mathrm{~m} / 2747 \mathrm{~m} \cdot \mathrm{s}^{-1}=51 \mu \mathrm{s}$ and a maximum pulse repetition frequency of $\mathrm{PRF}=1 / \mathrm{T}=19.5 \mathrm{kHz}$. Assuming an alternating operation of both arrays, in principle a maximum frame rate $\mathrm{f}_{\mathrm{r}}=\mathrm{PRF} / 2 \mathrm{n}=19.5 \mathrm{kHz} /(2 \cdot 6)=1.6 \mathrm{kHz}$ for the flow mapping can be achieved.

The parallel time division mode for one transducer line array is implemented by the control circuit according to Fig. 4. The measurement system operates as follows: An arbitrary function generator generates 8 wave cycles at $8 \mathrm{MHz}$ as excitation signal which is amplified to voltages around $50 \mathrm{~V}$ by a RF power amplifier. This high voltage signal is distributed by a transmitting multiplexer among the currently active transducers. The transmitting/receiving switch of every transducer element automatically toggles between transmitting and receiving. The received echo signals of the active transducers are directed to a receiving multiplexer distributing the echo signals among four summing amplifiers. These amplifiers sum the signals of the two piezo elements of a corresponding transducer as well as filters and amplifies the signal. A four-channel data aquisition card for Personal Computers digitizes and records the echo signals. The PC processes the recorded echo signals and determines the flow field by cross-correlation.

A microcontroller controls the transmitting and receiving multiplexer. Hence, it is triggered by the arbitrary function generator when an excitation signal is transmitted. Then the microcontroller calculates the next step of the time division scheme and switches the multiplexers [9].

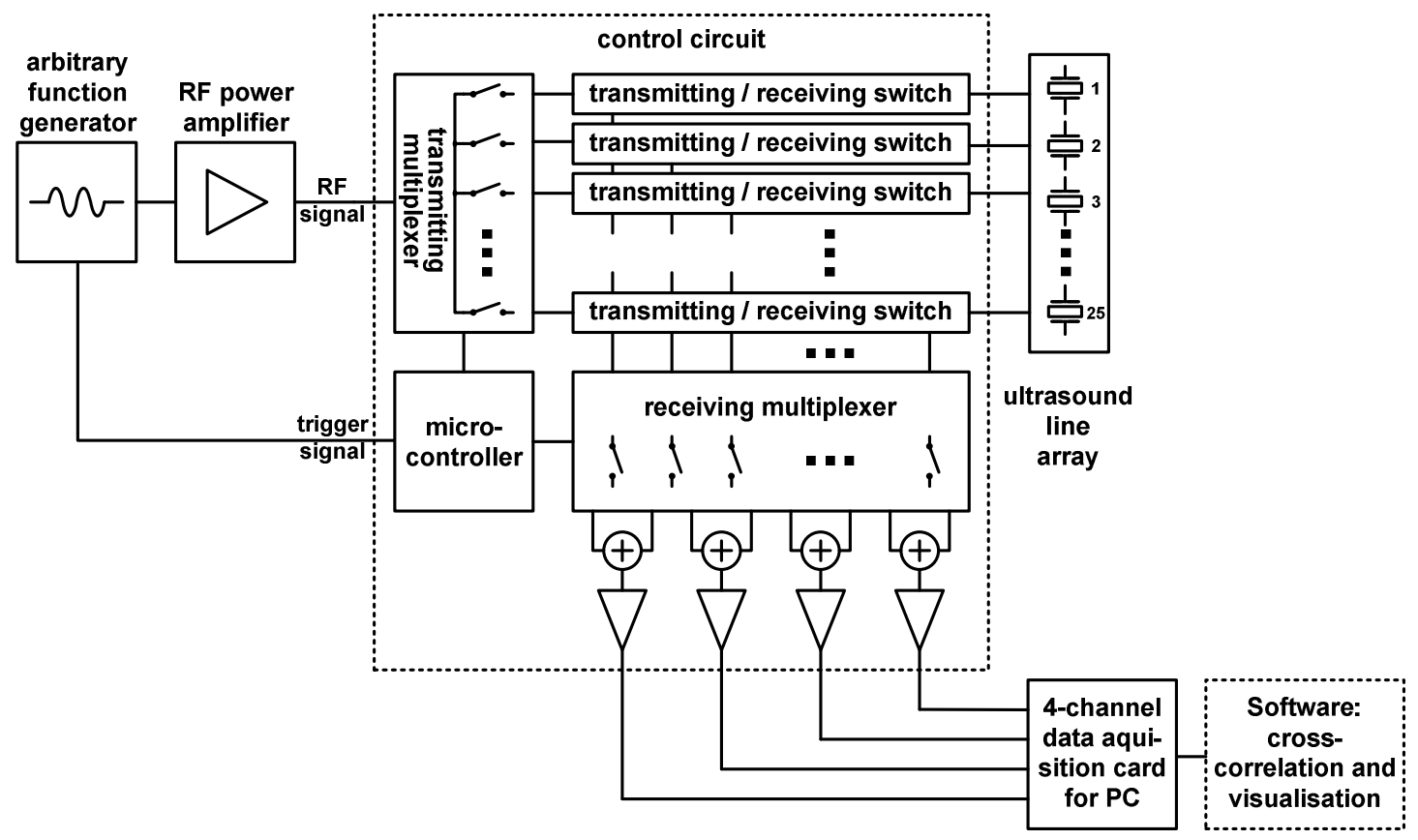

Figure 4. Measurement electronics with control circuit for generating the time division scheme 


\section{Flow Mapping}

To prove the functionality of the arrays first investigations were done with one transducer line array for $2 \mathrm{~d} / 1 \mathrm{c}$-measurements. Thereto, the liquid metal flow in a cubic vessel driven by a magnetic field was measured.

\subsection{Experimental Setup}

A magnetic field stirrer is used to drive the liquid metal flow. The stirrer consists of a system of induction coils (Fig. 5) generating a rotating magnetic field (RMF). Inside the magnetic stirrer a cubic vessel made of acrylic glass is placed centered. The vessel is filled with the liquid metal GalnSn. The ultrasound line array is clamped on one of the outer walls. That is, the flow is measured through the acrylic glass wall. For good coupling ultrasound gel is used.

The rotating magnetic field affects the liquid metal in the vessel as follows: The RMF rotates around the vertical axis of the vessel and induces electrical currents inside the liquid metal. The magnetic fields of these currents interact with the imposed magnetic field (Lorentz force). Thereby, a dominating angular component of the elctromagnetic force is generated which drives a swirling flow of the liquid metal. The angular velocity of the fluid is always smaller than the rotating field, because the induction of the electrical currents is based on a relative motion between field and fluid. A slip occurs similar to the principle of the asynchronous motor [10]. This swirling flow structures was measured with the ultrasound array measurement system and the results are discussed in the following.

Top view

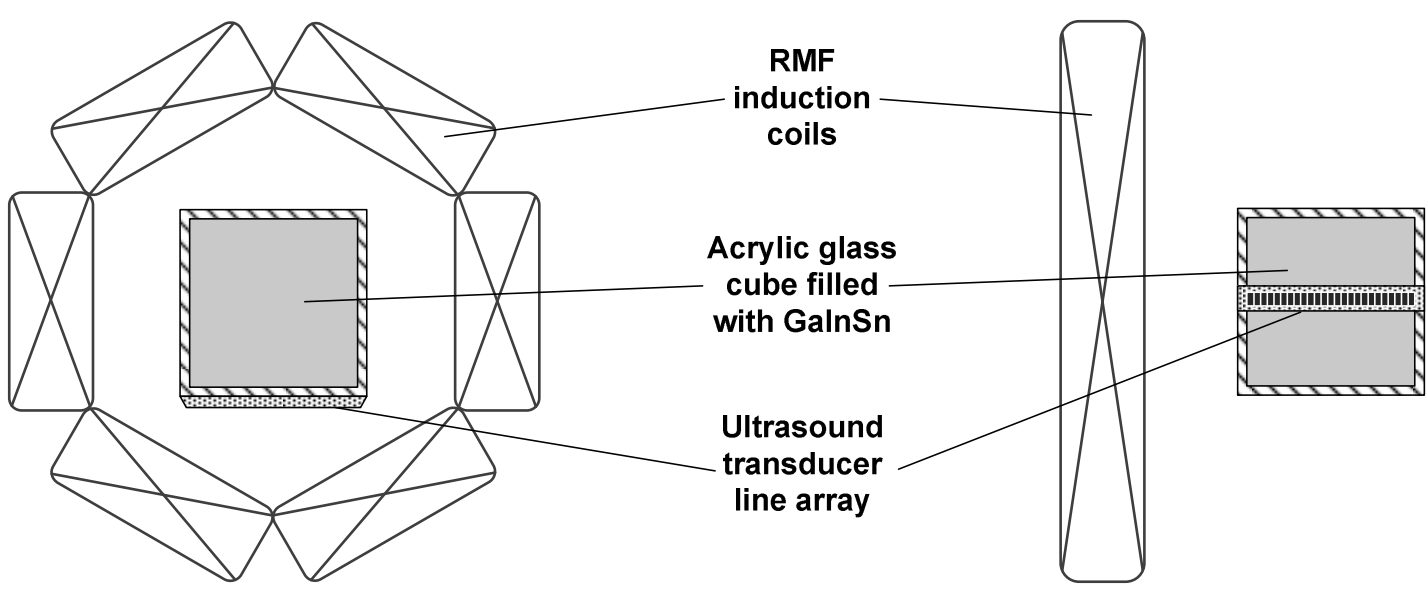

\section{Side view}

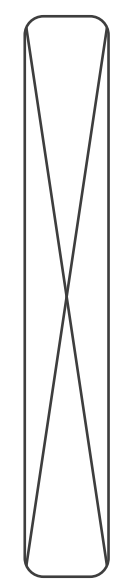

Figure 5. Experimental setup: Acrylic glass cube filled with GalnSn in a magnetic field stirrer

\subsection{Flow Measurement of Continuous RMF Driven Flow}

For measuring the swirling flow the transducer line array was installed at the outer wall of the vessel as shown in figure 6. The measurement plane conforms to the horizontal mid-plane of the cubic vessel.

The angular frequency of the rotating magnetic field amounts to $\omega=2 \pi \cdot 50 \mathrm{~Hz}=314 \mathrm{~Hz}$, the applied magnetic flux density to $B=4.2 \mathrm{mT}$. The result of this $2 \mathrm{~d} / 1 \mathrm{c}$ flow field measurement is shown in figure 7 . The transducer line array is placed along the $y$-axis and measures the velocity component of the flow in $x-$ direction. The nomenclature is such, that for positive velocities the flow is propagating away from the transducer. Thus the flow field in figure 7 characterizes a clockwise rotating flow (vortex) in GalnSn. Missing data points between two adjacent transducer profiles were interpolated.

Flow velocities up to $\mathrm{v}=114 \mathrm{~mm} \cdot \mathrm{s}^{-1}$ were measured resulting in a maximum angular frequency of $\omega_{\text {Flow }}=$ $\mathrm{v} / \mathrm{r}=114 \mathrm{~mm} \cdot \mathrm{s}^{-1} / 35 \mathrm{~mm}=3.26 \mathrm{~Hz}$. Therewith, the angular frequency of the RMF is around hundred times the angular frequency of the flow. The measurements show the slip between exciting RMF and driven flow. 


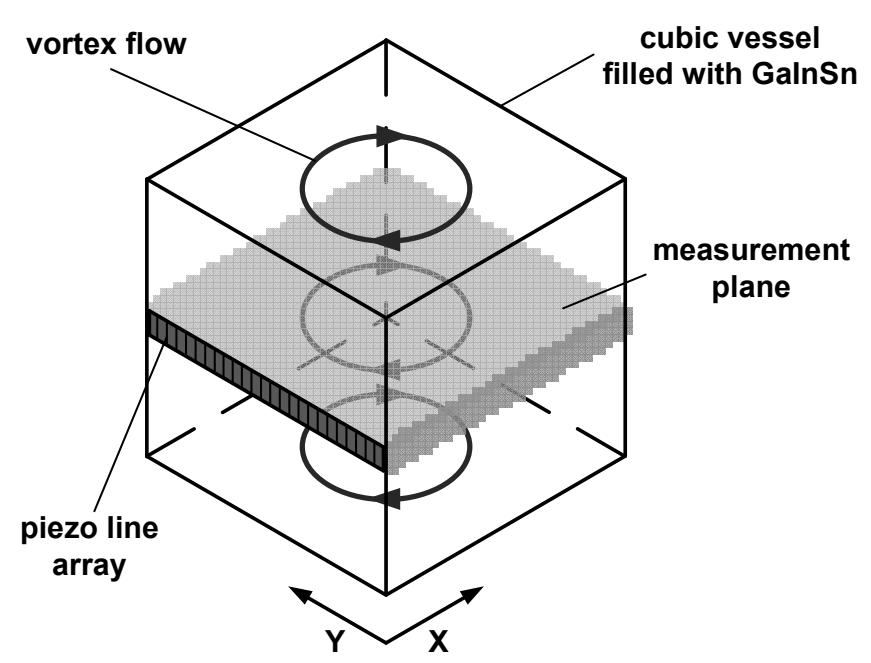

Figure 6. Measurement configuration for visualization of flow field $(2 \mathrm{~d} / 1 \mathrm{c})$ in the plotted measurement plane

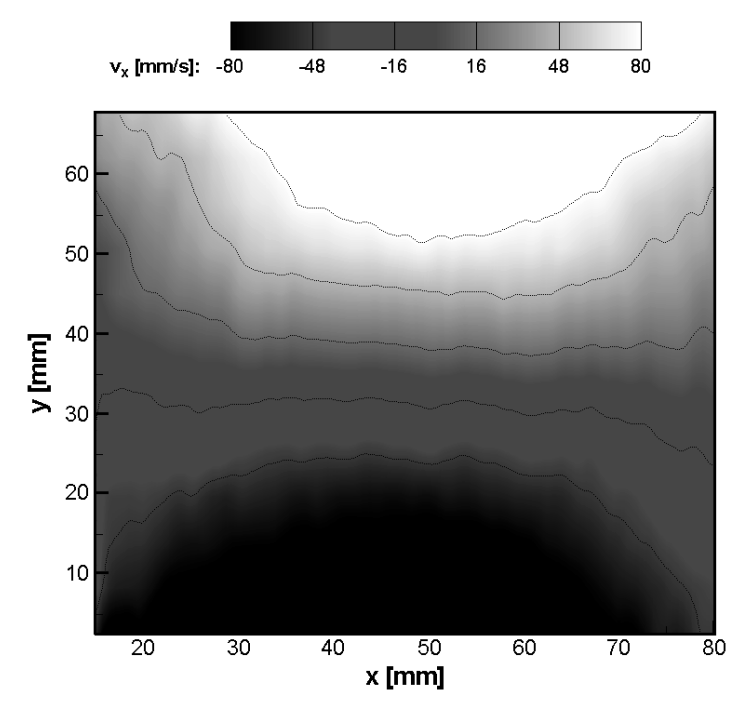

Figure 7. Vortex in clockwise direction; the velocity component in $\mathrm{x}$-direction is measured

\subsection{Flow Measurement of Pulsed RMF Driven Flow}

In magnetohydrodynamics not only continuous but also time-varying RMFs with reversal of flow direction are of interest. Hence, investigations were done with a RMF $\left(\omega=314 \mathrm{~Hz} ; \mathrm{B}_{0}=4.2 \mathrm{mT}\right)$ periodically changing its direction of rotation all 10 seconds (Fig. 8), e.g. $T=20 \mathrm{~s}$.

Figure 9 depicts the temporal sequence of one change of the rotating direction (i.e. a time span of $10 \mathrm{~s}$ ) of such a measurement. The first image of the flow mapping shows the moment when the rotating direction of the RMF changes $\left(t=t_{0}\right)$. Firstly, the outer vortex flow is slowed down, then the inner one. After about $t=t_{0}+3 \mathrm{~s}$ the outer flow has already reversed its rotation direction and is accelerated in turn while the inner flow is still rotating in the original direction (image 4 in Fig. 7). After the inner flow is slowed down completely (image 5 in Fig. 7), finally the inner flow also changes its flow direction. Now the rotation direction of the entire flow is reversed. Shortly before the direction of rotation alternates again the vortex achieves its full angular velocity.

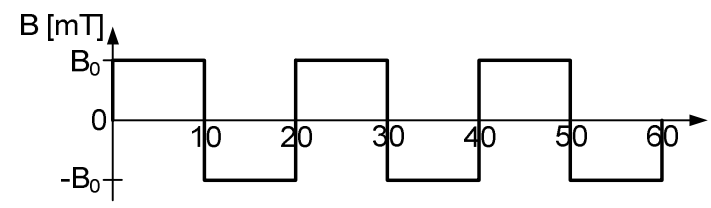

Figure 8. Periodical change of rotating direction of a rotating magnetic field

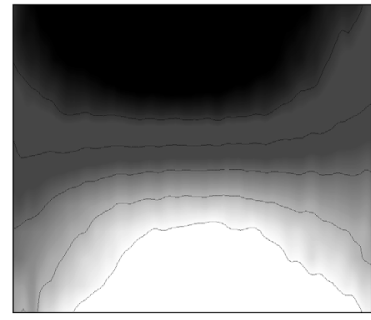

$t=t_{0}+0 s$

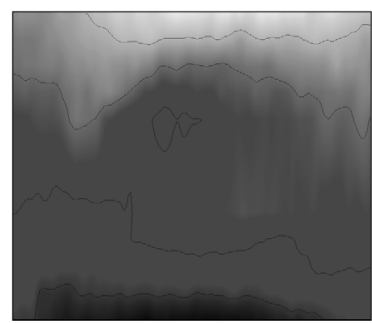

$\mathrm{t}=\mathrm{t}_{\mathrm{o}}+4 \mathrm{~s}$

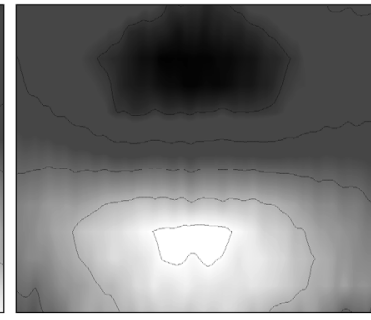

$\mathrm{t}=\mathrm{t}_{0}+1.75 \mathrm{~s}$

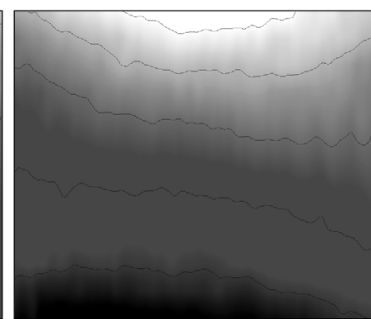

$\mathrm{t}=\mathrm{t}_{0}+6 \mathrm{~s}$

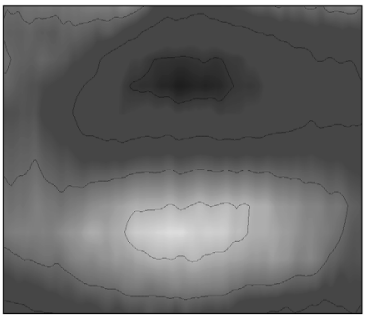

$\mathrm{t}=\mathrm{t}_{0}+2.5 \mathrm{~s}$

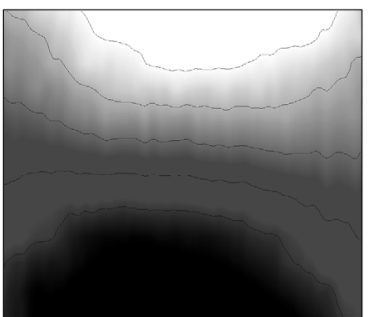

$\mathrm{t}=\mathrm{t}_{0}+8 \mathrm{~s}$

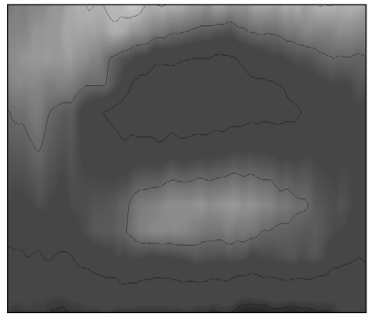

$\mathrm{t}=\mathrm{t}_{0}+3.25 \mathrm{~s}$

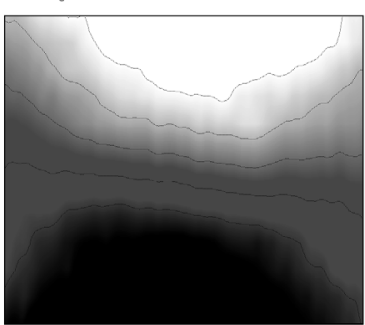

$\mathrm{t}=\mathbf{t}_{0}+10 \mathrm{~s}$ $v_{\mathrm{x}}[\mathrm{mm} / \mathrm{s}]$

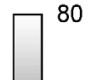

48

16

$-16$

$-48$

$-80$

Figure 9. Mapping of flow direction change in pulsed RMF within $10 \mathrm{~s}$; $t_{0}$ is the point of time when rotating direction of RMF changes from $-B_{0}$ to $B_{0}$ 


\section{Summary}

An ultrasound Doppler measurement system for evaluating flow fields in liquid metals has been presented. Two ultrasound transducer line arrays were employed arranged orthogonally to each other. Each array comprises 25 transducer elements and is driven by a special control scheme. In first measurements the operability of the measurement system in sequentially time division mode was proven. A cubic vessel filled with GalnSn was positioned in a rotational magnetic field (RMF) and the vortex flow induced by continuous RMF was visualized.

In the future the frame rate will be increased according to the presented scheme using parallel multiplexing. Then, a better characterization of swirling flows can be achieved. At prospective investigations both transducer line arrays will be applied in different environments for different flows and at different continuous or pulsed rotating magnetic fields for $2 \mathrm{~d} / 2 \mathrm{c}$-measurements.

\section{Acknowledgement}

The project workers thank the Deutsche Forschungsgemeinschaft (DFG) for the financial support of the project in context of the collaborative research center SFB 609 "Electromagnetic Flow Control in Metallurgy, Crystal Growth and Electrochemistry". Furthermore we thank Michael Lenz, Elfgard Kühnicke and Danilo Nicke for their assistance work as well as Dirk Räbiger and Thomas Gundrum and the other group members on ultrasound technique in the Forschungszentrum Rossendorf for their support. In particular we thank Dr. Richter from the company Richter Sensor \& Transducer Technologie who produced the ultrasound transducer line arrays.

\section{References}

[1] S. Eckert, G. Gerbeth, D. Räbiger, B. Willers, C. Zhang, "Experimental Modelling using Low Melting Point Metallic Melts: Relevance for Metallurgical Engineering'”, Steel Research International, vol. 78, no. 5, pp. 419-425, 2007.

[2] S. Eckert, P.A. Nikrityuk, D. Räbiger, K. Eckert, G. Gerbeth, "Efficient Melt Stirring Using Pulse Sequences of a Rotating Magnetic Field: Part I. Flow Field in a Liquid Metal Column", Metallurgical and Materials Transactions B, vol. 39B, pp. 375-386, 2008.

[3] M. Watanabe, M Eguchi, T. Hibiya, "Silicon Crystal Growth by the Electromagnetic Czochralski (EMCZ) Method", Japanese Journal of Applied Physics, vol. 38, no. 1A/B, pp. L10-L13, 1999.

[4] T. Toh, E. Takeuchi, "Electromagnetic Phenomena in Steel Continuous Casting", H. Aref \& J.W. Philips (Eds.), Mechanics for a New Millennium, Kluwer, pp. 99-112, 2001.

[5] J. Czarske, "Laser Doppler velocimetry using powerful solid-state light sources", Review Article, Measurement Science and Technology, vol. 17, pp. R71 - R91, 2006.

[6] Signal Processing SA, Switzerland, www.signal-processing.com

[7] A. Cramer, C. Zhang, S. Eckert, "Local flow structures in liquid metals measured by ultrasonic Doppler velocimetry", Flow Measurement and Instrumentation, vol. 15, pp. 145-153, 2004.

[8] Wayne R. Hedrick, David L. Hykes, Dale E. Starchman, Ultrasound Physics and Instrumentation. ELSEVIER MOSBY, 4 ed., 2005.

[9] S. Franke, L. Büttner, J. Czarske, S. Eckert, G. Gerbeth, „Messung der Strömungsgeschwindigkeits-verteilung in Flüssigmetallen mittels eines Ultraschall-Doppler-ArraySystems“, XXII. Messtechnisches Symposium des Arbeitskreises der Hochschullehrer für Messtechnik e.V., September 11-13, 2008, Dresden, pp. 217-229.

[10] P. A. Davidson, J. C. R. Hunt, "Swirling recirculating flow in a liquid-metal column generated by a rotating magnetic field", Journal of Fluid Mechanics, vol. 185, pp. 67-106, 1987. 